\title{
LIF-Induced STAT3 Signaling in Murine versus Human Embryonal Carcinoma (EC) Cells
}

\author{
J an J acob Schuringa,*'† Saskia van der Schaaf,* Edo Vellenga,† Bart J . L. Eggen,*,1 and Wiebe Kruijer* \\ *Department of Developmental Genetics, Biological Center, Kerklaan 30, 9751 NN Haren, The Netherlands; and \\ †Department of Hematology, University Hospital Groningen, Hanzeplein 1, 9700 RB Groningen, The Netherlands
}

\begin{abstract}
Self-renewal and the maintenance of pluripotency of mouse embryonal stem (ES) cells in vitro requires exogenous leukemia inhibitory factor (LIF). Mouse ES cells can be cultured and kept undifferentiated in the absence of embryonal feeder-cell layers when exogenous LIF concentrations are maintained above a threshold concentration. An important downstream target of LIF signal transduction in mouse ES cells is the transcription factor signal transducer and activator of transcription 3 (STAT3). In contrast to mouse ES cells, human ES cells are unresponsive to LIF and depend on feeder cells for undifferentiated growth. Here, we investigated the activation patterns of LIFdownstream effectors in mouse and human embryonal carcinoma (EC) cells. We report that LIF induces both ERK-1 as well as STAT3 activation in mouse P19 EC cells. LIF enhances the proliferation rate of P19 EC cells, which depends on ERK activity but does not require activation of STAT3. In contrast, LIF does not activate STAT3, ERK, or the gp130 receptor in human $\mathrm{N}$ tera-2/D1 E C cells, although all receptor components are expressed. The negative feedback protein suppressor of cytokine signaling 1 (SOCS-1) is constitutively expressed in $\mathrm{N}$ tera-2/D1 EC cells, suggesting that LIF signal transduction is inhibited by elevated levels of SOCS-1 expression. ๑ 2002 Elsevier Science (USA)
\end{abstract}

Key Words: LIF; STAT3; embryonal carcinoma; P19 EC; N tera-2/D1 EC.

\section{INTRODUCTION}

Embryonal stem (ES) ${ }^{2}$ cells are pluripotent cells derived from the inner cell mass of preimplantation em-

\footnotetext{
${ }^{1}$ To whom correspondence and reprint requests should be addressed. Fax: (+31)50-3632348. E-mail: b.j.l.eggen@biol.rug.nl.

${ }^{2}$ Abbreviations used: STAT, signal transducer and activator of transcription; SOCS, suppressor of cytokine signaling; LIF, leukemia inhibitory factor; hLIF, human LIF ; mLIF, murine LIF ; IL-6, interleukin-6; ERK, extracellular regulated kinase; EC cell, embryonal carcinoma cell; ES cell, embryonal stem cell; LIFR, LIF receptor; J AK, J anus kinase; DMEM, Dulbecco's modified Eagle's medium; FCS, fetal calf serum; IRE, IL-6 response element; PBS, phosphatebuffered saline; LUC, luciferase.
}

bryos [1, 2], which differentiate into specific cell types that comprise all the three germ-layers of the embryo $[3,4]$. Mouse ES cells can be maintained in an undifferentiated state when cultured on fibroblast feeder cell layers or in the presence of leukemia inhibitory factor (LIF) on gelatin-coated tissue culture dishes [5-8]. LIF belongs to the interleukin (IL)-6 family of cytokines, which also includes oncostatin $M$, ciliary neurotropic factor, IL-11, cardiotrophin, and IL-6 [9]. The IL-6-type cytokines all utilize the common gp130 receptor chain, and LIF signaling is mediated through the LIF receptor (LIFR- $\beta$ ) which heterodimerizes with the gp130 receptor upon LIF binding [10]. Activation of the LIFR- $\beta$-gp130 heterodimer results in the rapid activation of J anus kinases (J AKs) which in turn phosphorylate tyrosine residues of LIFR- $\beta$ and gp130 [9, 11]. These phosphorylated tyrosine residues form docking sites for signaling molecules including STAT3 and SHP2 [9]. STATs are transcription factors, which form dimers upon phosphorylation of a specific tyrosine residue that is located in a conserved $\mathrm{SH} 2$ domain [12]. STAT dimerization allows nuclear translocation and the transcriptional activation of target genes [12]. SHP2 is a tyrosine phosphatase which signals upstream of the Ras/MAP kinase signal transduction pathway [9, 13-15]. Recently, a family of cytokineinducible inhibitors of signaling has been identified that downregulate the $\mathrm{J}$ ak/STAT signaling pathway [16-18]. The proteins in this family, including cytokine-inducibleSH-2-containing protein and suppressor of cytokine signaling (SOCS)/] ak-binding protein/ STAT-induced STAT inhibitor proteins, are proteins containing $\mathrm{SH} 2$ domains which interact with J AKs, thus preventing the activation of STATs [16-18]. Specifically, SOCS-1 and -3 are implicated in the downregulation of the IL-6-induced activation of STAT3 [19-22]. Moreover, SOCS-1 and SOCS-3 can quickly be upregulated by IL-6 [17].

While embryonal stem cells are derived from the inner cell mass of the blastocyst stage of the embryo, embryonal carcinoma (EC) cells are the stem cells of teratocarcinomas induced by transplantation of preimplantation embryos or ES cells to extrauterine sites [4, 
23, 24]. Human EC cells have been isolated from testicular germ cell tumors and resemble human embryonal stem cells with regard to marker expression [23, 24]. Both murine and human EC cells can be cultured on gelatin coated tissue culture dishes and can be differentiated into specific cell types of all three germ layers upon treatment with the appropriate growth factors or cytokines $[23,25,26]$.

The pluripotency of mouse stem cells in culture depends on LIF-activated STAT3, since mutation of either the gp130 receptor or STAT3 itself abrogated the self-renewal of ES cells and led to the onset of differentiation $[5,7,8]$. Consequently, mouse stem cells can be cultured and kept pluripotent on gelatin-coated tissue culture flasks when maintained in medium containing LIF. In contrast, human stem cells in culture depend on feeder cells in order to maintain their pluripotency [27]. In the experiments presented here, it was investigated which signal transduction cascades are activated in response to LIF in human N tera-2/D1 EC versus murine P19 EC cells. We conclude that LIF induces both ERK and STAT3 activation in mouse EC cells, but that LIF-induced proliferation depends only on ERK activity. In contrast, LIF does not activate STAT3 or ERK-1 in human EC cells, although all receptor components are expressed. Constitutive SOCS-1 expression and lack of gp130 activation in response to LIF are observed, suggesting that STAT3 signaling in human $\mathrm{N}$ tera-2/D1 cells is prevented by el evated levels of SOCS-1.

\section{MATERIALS AND METHODS}

Cell culture, reagents, and antibodies. The murine teratocarcinoma cell line P19 EC and the human teratocarcinoma cell line N tera-2/D1 EC were cultured in Dulbecco's modified Eagle's medium (DMEM)/HAM F-12 medium supplemented with $7.5 \%$ heat-inactivated fetal calf serum (FCS, Integro B.V., Zaandam, The Netherlands). The human hepatoma cell line HepG2 was grown in DMEM supplemented with $10 \%$ FCS. Cells were stimulated with $10^{6}$ units/ml murine LIF (ESGRO) or human LIF (obtained from Life Science and Sigma, respectively) or with $25 \mathrm{ng} / \mathrm{ml}$ human recombinant IL-6 (generous gift from Dr. S. C. Clark, Genetics Institute, Cambridge, MA). The MEK inhibitor PD98059 and the J AK2 inhibitor AG490 (both obtained from Calbiochem) were used at final concentrations of $2050 \mu \mathrm{M}$, respectively. Antibodies against hemagglutinin STAT3, gp130, and ERK-1 (Santa Cruz) were used in dilutions of 1:3000, unless stated otherwise. Antibodies against phosphorylated STAT3 (tyr705), STAT3 (ser727), and ERK p44/p42 (thr202/tyr204) were obtained from New England Biolabs and used in a 1:1000 dilution. Antibodies against phospho-tyrosine (PY-99) were obtained from Santa Cruz and were used in a 1:1000 dilution.

Expression and reporter constructs. The following plasmids were used: pIRE LUC containing two copies of the IL-6 response element (plRE) of the ICAM-1 promoter in front of the Herpes simplex virus thymidine kinase promoter and the luciferase gene and in the pIREmut-luc reporter the STAT3 binding sites were mutated [28]. The pl C-1014-LU C reporter containing a 1014-bp fragment of the human ICAM-1 promoter and the plC-1014(IRE-mut)-LUC reporter in which the IRE site was mutated were previously described [29]; pSG5-STAT3 which expresses STAT3 from the SV40 promoter (gen- erous gift from Dr. P. Coffer, Department of Pulmonary Diseases, AZU, Utrecht, The Netherlands); pSG5-STAT3 $\beta$, which expresses a dominant negative isoform of STAT3 lacking the 55 C-terminal amino-acid residues (generous gift from Dr. R. de Groot, Department of Pulmonary Diseases, AZU); pCMV-MEN2A-RET(634), which expresses the Cys634 mutant of RET [30]; and pCMV-JAK1 and pCMV-J AK2 expressing J AK 1 and J AK 2 from the CMV promoter, respectively (generous gifts from Dr. D. Levy, Department of Pathology, School of Medicine, NYU, New York, NY).

Transient transfections. $\mathrm{N}$ tera-2/D1 EC and P19 EC cells were seeded at $1 \times 10^{5}$ cells per well and pHepG 2 cells were seeded at $3 \times$ $10^{5}$ cells per well in six-well plates (Costar), and $24 \mathrm{~h}$ later cells were transfected with $10 \mu \mathrm{g}$ plasmid DNA using the calcium phosphate coprecipitation method [31]. Transfection mixtures consisted of a mixture of $3 \mu \mathrm{g}$ plRE LUC reporter, $3 \mu \mathrm{g}$ pDM2-LacZ as a control to determine transfection efficiency, and $2.5 \mu \mathrm{g}$ of expression plasmids for dominant negative or constitutively active signal transduction components unless stated otherwise under Results section. When necessary, pUC18 was added to the transfection mixture to obtain a total of $10 \mu \mathrm{g}$ of DNA. Cells were incubated with precipitate for $24 \mathrm{~h}$, washed with phosphate-buffered saline (PBS), and stimulated for an additional $24 \mathrm{~h}$. Cells were collected in $200 \mu \mathrm{l}$ reporter lysis buffer (Promega) and subjected to the assays for luciferase [32] and $\beta$-galactosidase [33] as previously described. The data represent two independent experiments using different batches of DNA, and in each experiment transient transfections were performed in triplicate. Standard deviations were calculated using Sigmaplot ( andel Corp.).

SDS-polyacrylamide ge el ectrophoresis, Western blotting, and immunoprecipitations. A total of $1 \times 10^{7}$ cells were lysed on ice in lysis buffer (20 mM Hepes pH 7.4, 2 mM EGTA, 1 mM DTT, 1 mM Na $\mathrm{NO}_{3}$ (ortho), 1\% Triton X-100, 10\% glycerol, $10 \mu \mathrm{g} / \mathrm{ml}$ leupeptin, and 0.4 mM PMSF). Prior to SDS-polyacrylamide gel electrophoresis, protein concentrations were determined (Bio-Rad), and equal amounts were used in the experiments. Whole-cell extracts were boiled for 5 min in the presence of Laemmli sample buffer prior to separation on $12.5 \%$ SDS-polyacrylamide gels. The proteins were transferred to a nitrocellulose filter (Millipore) in Tris-glycine buffer at $100 \mathrm{~V}$ for $1.5 \mathrm{~h}$ using an electroblotter (Pharmacia). Membranes were blocked with PBS buffer containing 5\% nonfat milk prior to incubation with antibodies. Binding of each antibody was detected by chemiluminesence using ECL according to the manufacturer's recommendations (Amersham Corp.). For immunoprecipitations, whole-cell lysates were incubated with anti-gp130 antibodies, precipitated with protein A-Sepharose beads (Pharmacia), and washed three times with Iysis buffer. The precipitates were boiled for $5 \mathrm{~min}$ in Laemmli sample buffer and subjected to $12.5 \%$ SDS-polyacrylamide gel electrophoresis.

Proliferation assays. For proliferation assays, $2500 \mathrm{~N}$ tera-2/D1 EC or P19 EC cells were cultured in 96-well plates, and proliferation of each cell line was followed for 5 days using an MTC assay according to the manufacturer's recommendations (Promega).

RNA extraction and RT-PCR. For RT-PCR, total RNA was isolated from $10^{6}$ cells using Trizol according to the manufacturer's recommendations (Life Technologies). $3 \mu \mathrm{g}$ of RNA per sample was reverse transcribed with $M-M u L V$ reverse transcriptase (Boehringer Mannheim). For PCR, $2 \mu \mathrm{l}$ of cDNA was amplificated using mouse gp80 primers (forward, 5'-CCAACCACGAAGGCTGTGCT-3'; reverse, 5'-GCTCCACTGGCCAAGGTCAA-3'), mouse gp130 primers (forward, 5'-CCACATACGAAGACAGACCA-3'; reverse, 5'-GCGTTCTCTGACAACACACA-3'), mouse LIF-R primers (forward, 5'-CAACCAACAACATGCGAGTG-3'; reverse, 5'-GGTATTGCCGATCTGTCCTG-3'), GAPDH primers (forward, 5' -ATCACCATCTTCCAGGAG-3'; reverse, 5'-GCCATCCACAGTCTT-3'), $\beta$-2-globulin primers (forward, 5'-CCAGCAGAGAATGGAAAGTC-3'; reverse, 5'-GATGCTGCTTACATGTCTCG), SOCS-1 primers (forward, 5'-CACGCACTTC- 
A

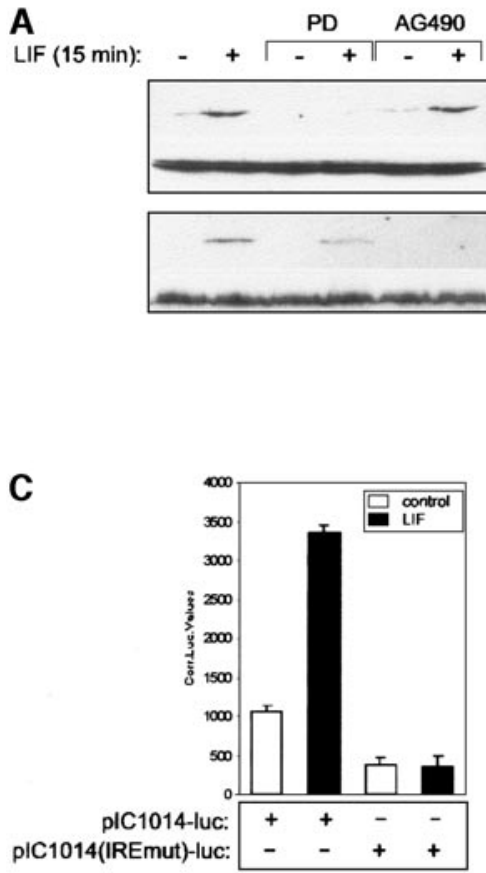

B

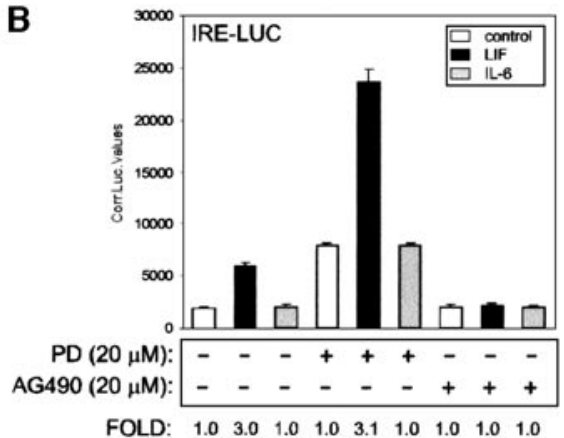

D

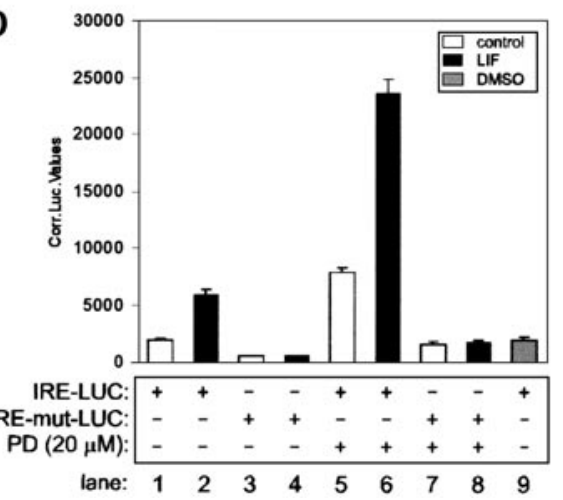

FIG. 1. LIF induces activation of ERK and STAT3 in mouse P19 EC cells. (A) P19 EC cells were treated with the MEK-1 inhibitor PD98059 $(20 \mu \mathrm{M})$ or the J AK-2 inhibitor AG490 $(50 \mu \mathrm{M})$ for $30 \mathrm{~min}$ as indicated prior to stimulation with $10^{6}$ units/ml murine LIF for 15 min. Total cell extracts were Western blotted using antibodies recognizing phosphorylated ERK p44/p42 (thr202/tyr204), phosphorylated STAT3 (tyr705), or unphosphorylated STAT3 or ERK p44/p42. (B) P19 EC cells were transiently transfected with the IRE-LUC reporter, pretreated with PD98059 $(20 \mu \mathrm{M})$ or AG490 $(50 \mu \mathrm{M})$ for $30 \mathrm{~min}$ as indicated, and stimulated with $10^{6} \mathrm{units} / \mathrm{ml}$ murine LIF for an additional $24 \mathrm{~h}$. Cells were harvested and lysates were used for luciferase and LacZ assays. Data represent average values of at least three independent experiments and each experiment was performed in triplicate. (C) Transient transfected assays as in B, but now cells were transfected with the ICAM-LUC reporter (pl C1014-Iuc) or the ICAM-LUC reporter in which the STAT3 binding sites were mutated (pl C1014(IRE mut)-luc). (D) Transient transfection assays as in B, using the IRE-LUC and IRE-mut-LUC reporters.

CGCACATTCC-3'; reverse, 5'-TCCAGCAGCTCGAAGAGGCA-3' ), or SOCS-3 primers (forward, 5'-TCACCCACAGCAAGTTTCCCGC-3'; reverse, 5'-GTTGACGGTCTTCCGACAGAGATGC-3') in a total volume of $50 \mu \mathrm{l}$ using 2 units of Taq polymerase (Boehringer Mannheim). After 25 cycles, 15- $\mu$ l aliquots were run on $1.5 \%$ agarose gels.

\section{RESULTS}

\section{LIF Induces both STAT3 and ERK-1 Activation in Murine P19 EC Cells}

To determine whether STAT3 and ERK are activated in response to LIF stimulation in P19 EC cells, total lysates were Western blotted using antibodies recognizing phosphorylated STAT3 (tyr 705) and ERK p44/p42 (thr202/tyr204). As depicted in Fig. 1A, 15 min of LIF stimulation induced both STAT3 and ERK-1 activation. LIF-induced activation of STAT3 and ERK-1 correlated with a cytoplasmic-nuclear translocation of both proteins as determined by immunofluorescence microscopy (data not shown). Pretreatment with the MEK-1 inhibitor PD98059 completely abol- ished the LIF-induced ERK-1 phosphorylation, while STAT3 phosphorylation was not altered (Fig. 1A). Pretreatment with the JAK-2 inhibitor AG490 did not affect LIF-induced ERK-1 phosphorylation, but STAT3 phosphorylation upon LIF stimulation was completely abolished, indicating that J AK-2 is the major tyrosine kinase that phosphorylates STAT3 on tyr705 in response to LIF in P19 EC cells (Fig. 1A).

STAT3 transactivation was determined by transiently transfecting P19 EC cells with the synthetic IRE-luciferase reporter (IRE-LUC), which contains two STAT3 binding sites in the promoter. LIF induced a three-fold induction of STAT3 transactivation, while IL-6 did not activate the IRE reporter (Fig. 1B). Inhibition of ERK activity using the inhibitor PD98059 did not affect the LIF-induced STAT3 transactivation, which was still approximately threefold, although both control and LIF-induced luciferase values were significantly higher compared to cells that were not treated with PD98059 (Fig. 1B). Inhibition of J AK-2 kinase activity using the inhibitor AG490 completely abol- 
A

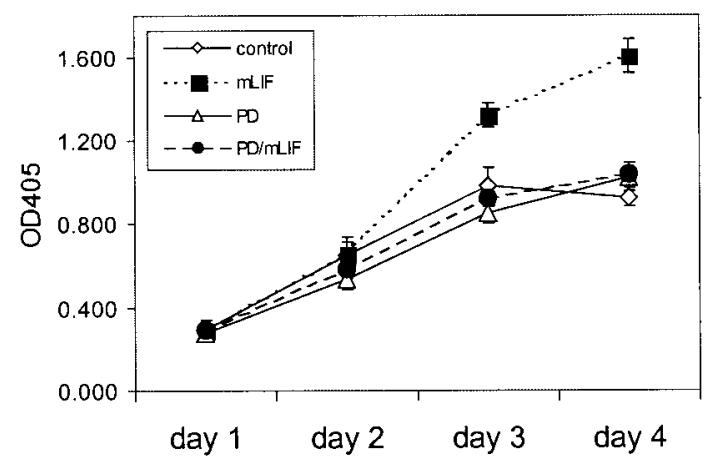

$\mathbf{B}$

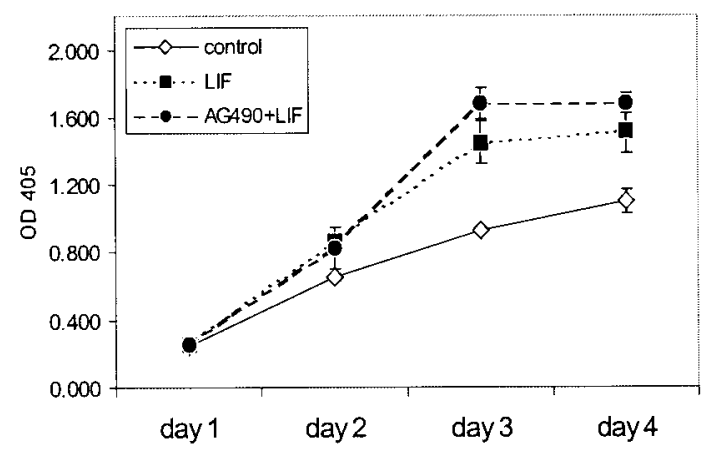

FIG. 2. LIF-induced proliferation of $P 19 E C$ cells depends on ERK activity but does not require activation of STAT3. (A) The proliferation of P19 EC cells was followed during 4 days using MTC assays. Cells were pretreated with the MEK-1 inhibitor PD98059 (20 $\mu$ M) as indicated for $30 \mathrm{~min}$ prior to stimulation with $10^{6}$ units/ml murine LIF at day 1 . (B) MTC assay as in A, but now cells were pretreated with the J AK-2 inhibitor AG490 (50 $\mu \mathrm{M})$ as indicated for 30 min prior to stimulation with murine LIF.

ished STAT3 transactivation in response to LIF (Fig. 1B), in agreement with the observed effects of AG490 on LIF-induced STAT3 tyr705 phosphorylation. Furthermore, we investigated the effects of LIF-induced STAT3 transactivation on natural promoters. As depicted in Fig. 1C, LIF induced a strong activation of the human ICAM-1 promoter (plC1014-luc), which was mediated via STAT3 since a reporter that contains the ICAM promoter in which the STAT3 binding sites were mutated (plC1014(IREmut)-luc) was not activated upon LIF stimulation.

To further study the effects of PD98059 on LIFinduced STAT3 transactivation, the IRE-mut-LUC reporter, in which the STAT3 binding sites were mutated, was transiently transfected in P19 EC cells. As depicted in Fig. 1D, LIF did not activate the IRE-mutLUC reporter, while the enhancing effects of PD98059 were observed on both the IRE-LUC and the IRE-mutLUC reporters (compare lanes 1 and 2 with 5 and 6 and lanes 3 and 4 with 7 and 8 ). The enhancing effects of PD98059 on transcriptional activities were also observed on various other promoters, including the ICAM and cyclin D1 promoters (data not shown), indicating that inhibition of ERK activity does not directly affect STAT3 signal transduction but rather positively affects gene transcription in a general manner in P19 EC cells.

Taken together, these data indicate that LIF induces both STAT3 and ERK-1 activation in P19 EC cells while AG490 and PD98059 serve as specific inhibitors for STAT3 and ERK, respectively.

\section{LIF-Induced Proliferation of P19 EC Cells Involves Activation of ERK, but N ot of STAT3}

Since LIF-induced activation of STAT3 is required for murine stem cell renewal and the maintenance of their pluripotency, the role of LIF and STAT3 for P19
EC proliferation was investigated. LIF stimulation resulted in an approximately twofold increase in P19 EC cell proliferation (Fig. 2A). Inhibition of ERK activity using PD98059 completely abolished the LIF-induced proliferation, while proliferation rates in the absence of LIF stimulation remained unaffected. Treatment with the JAK-2 inhibitor AG490 did not affect the LIFinduced proliferation of P19 EC cells (Fig. 2B). These data indicate that LIF-induced P19 EC proliferation requires ERK activation but not STAT3 activation (Fig. 2B).

\section{LIF Does Not Activate STAT3 or ERK in Human N \\ Tera-2/ D1 EC Cells}

Next, it was investigated whether LIF and I L-6 activate STAT3 and ERK in the human embryonal teratocarinoma cell line N tera-2/D1 EC. Total extracts of unstimulated or stimulated cells were Western blotted using antibodies recognizing phosphorylated STAT3 (tyr705) and ERK p44/p42 (thr202/tyr204). As depicted in Fig. 3A, no STAT3 tyr705 phosphorylation was observed in response to either LIF or IL-6, while STAT3 was highly expressed. Furthermore, high basal levels of ERK-1 phosphorylation were observed in N tera2/D1 EC cells, which were not further upregulated upon LIF stimulation (Fig. 3B). As expected, no LIF - or IL-6-induced STAT3 transactivation was observed in transient transfection assays using the IRE-Iuciferase reporter (Fig. 3C) or the ICAM-luciferase reporter (data not shown). Inhibition of ERK activity by treatment with PD98059 did not restore the STAT3 inducibility by either LIF or IL-6, but higher basal levels of reporter activity were observed (Fig. 3C), as was the case in P19 EC cells. Since murine LIF was used in previous experiments, we determined whether human LIF could activate STAT3 in the human cell line N tera-2/D1 EC. As depicted in Fig. 3D, neither murine 
A

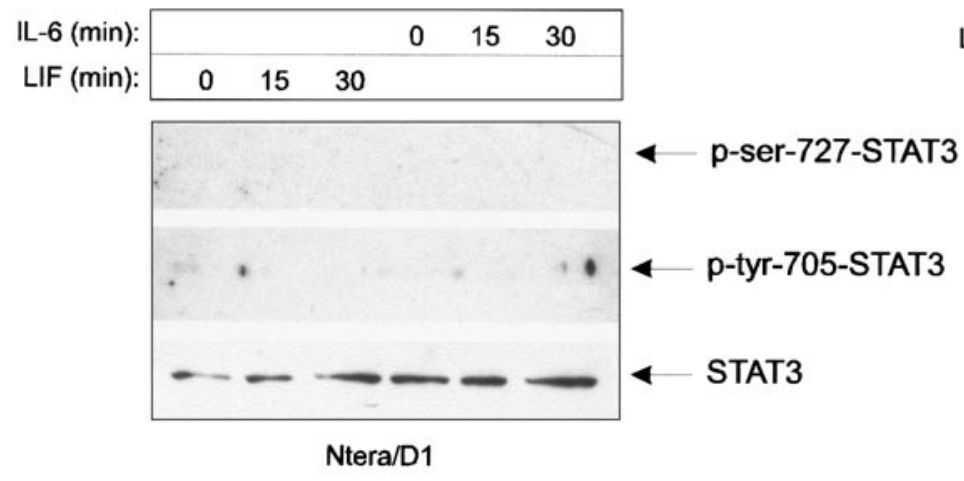

B

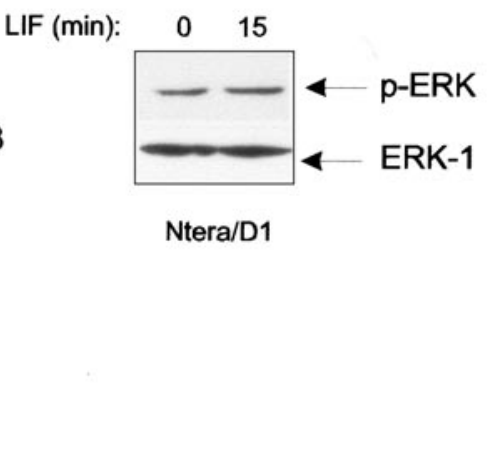

C

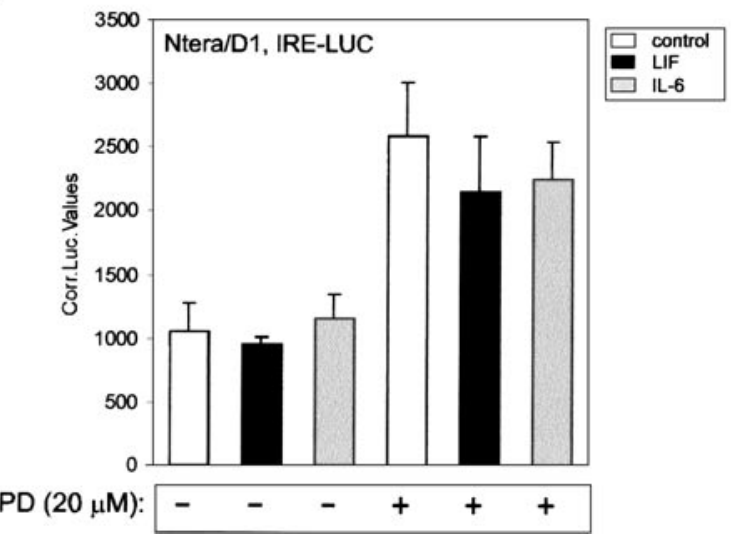

D

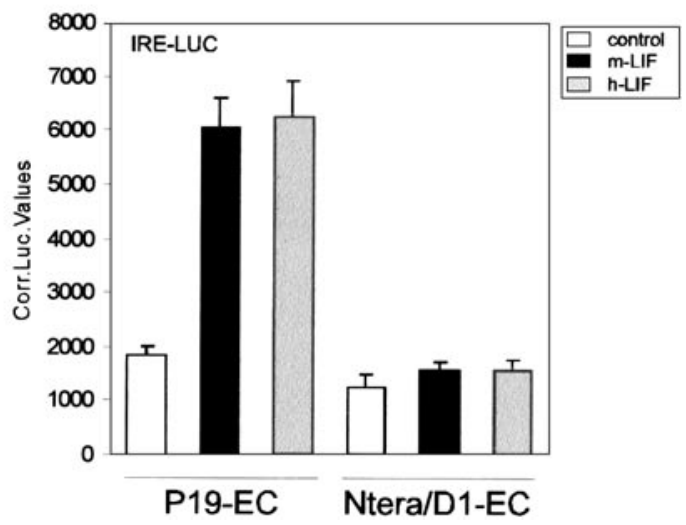

FIG. 3. LIF and IL-6 do not activate STAT3 or ERK in human $\mathrm{N}$ tera-2/D1 EC cells. N tera-2/D1 EC cells were stimulated with $25 \mathrm{ng} / \mathrm{ml}$ human IL-6 or $10^{6}$ units/ml murine LIF for the indicated time-periods. Total cell extracts were Western blotted using antibodies recognizing STAT3 or phosphorylated STAT3 (tyr705) (A), or ERK p44/42 or phosphorylated ERK p44/p42 (thr202/tyr204) (B). (C) N tera-2/D1 EC cells were transiently transfected with the IRE-LUC reporter, pretreated with PD98059 $(20 \mu \mathrm{M})$ for $30 \mathrm{~min}$ as indicated and stimulated with $10^{6}$ units/ml murine LIF for an additional $24 \mathrm{~h}$. Cells were harvested and lysates were used for luciferase and LacZ assays. (D) Transient transfection assay as in C using P19 EC or N tera-2/D1 EC cells as indicated, but now cells were stimulated with either $10^{6}$ units/ml murine LIF or $10^{6}$ units/ml human LIF.

LIF nor human LIF induced STAT3 transactivation in $\mathrm{N}$ tera-2/D1 EC cells, while both murine and human LIF induced STAT3 transactivation in P19 EC cells to comparable levels. Taken together, these data indicate that neither LIF nor IL-6 can activate STAT3 or ERK in $\mathrm{N}$ tera-2/D1 EC cells.

\section{LIF Does Not Induce Proliferation of Human N}

Tera-2/D1 EC Cells

To determine whether LIF induces proliferation of $\mathrm{N}$ tera-2/D1 EC cells MTC assays were performed using unstimulated as well as hLIF-stimulated cells. As depicted in Fig. 4A, hLIF did not enhance $\mathrm{N}$ tera-2/D1 EC proliferation over basal proliferation levels. Inhibition of ERK activity using PD98059 reduced the proliferation rates of unstimulated cells, while still no effects of hLIF stimulation were observed in the presence of PD98059 (Fig. 4A). Since cells were cultured in 7.5\% FCS, which al ready induced a significant proliferation, it was investigated whether LIF could induce $\mathrm{N}$ tera2/D1 EC proliferation at lower serum concentrations. As depicted in Fig. 4B, no serum-induced proliferation was observed in the presence of 0.5 or $0.1 \% \mathrm{FCS}$, while LIF stimulation did not enhance proliferation rates. These data indicate that LIF does not induce proliferation of human teratocarcinoma $\mathrm{N}$ tera-2/D1 EC cells as has also been reported for human stem cells.

Lack of LIF Signaling in Human N Tera-2/D1 EC

Cells Is Not Caused by an Aberrant Expression

of Required Signal Transduction Components

Since no effects of LIF were observed on $N$ tera-2/D1 proliferation rates and on STAT3 and ERK activation in these cells, it was investigated whether all the required signal transduction components are expressed in $\mathrm{N}$ tera-2/D1 cells. Total RNA was isolated from $\mathrm{N}$ tera-2/D1 EC cells from which CDNA was prepared. PCRs were performed using primers for the IL -6 and 
A

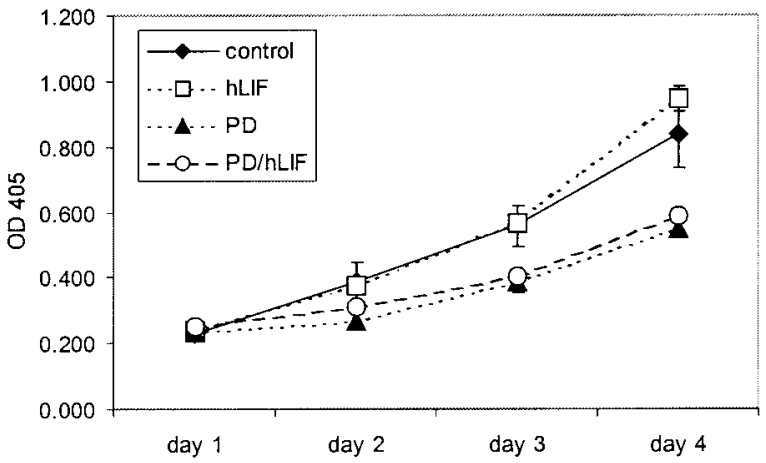

B

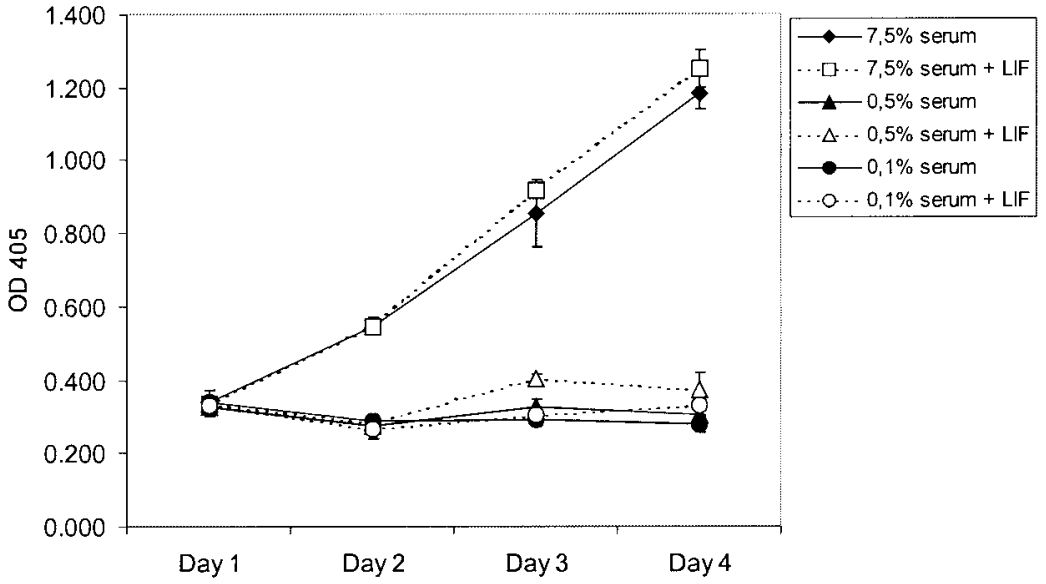

FIG. 4. LIF does not induce proliferation of $N$ tera-2/D1 EC cells. (A) The proliferation of $N$ tera-2/D1 EC cells was followed for 4 days using MTC assays. Cells were grown in 7.5\% FCS and pretreated with the MEK-1 inhibitor PD98059 (20 $\mu$ M) as indicated for 30 min prior to stimulation with $10^{6}$ units/ml human LIF at day 1 . (B) MTC assays as in A, but now cells were grown in $7.5,0.5$, or $0.1 \% \mathrm{FCS}$ as indicated.

LIF receptor components gp80, gp130. and LIF-R. As depicted in Fig. 5A, all the receptor components which are required for LIF and IL-6 signaling are expressed in N tera-2/D1 EC cells. As a control, RNA isolated from murine P19 EC was used for RT-PCR reactions. In P19 EC cells, the gp130 and LIF-R components are expressed, while no gp80 expression was observed (Fig. $5 A$ ). These data suggest that IL-6 does not activate STAT3 in P19 EC cells due to a lack of gp80 expression, which is required to mediate IL-6 signal transduction. Gp130 expression was also verified in both N tera-2/D1 EC and P19 EC cells by Western blotting (Fig. 5B).

To investigate whether J AK or STAT3 expression is disturbed in N tera-2/D1 EC cells, the IRE-LUC re porter was transiently transfected together with expression vectors for J AK-1, J AK-2, or STAT3. hLIF did not induce STAT3 transactivation in the presence of overexpressed exogenous J AK-1, J AK-2, or STAT3, although basal IRE transactivation levels were enhanced when both JAK-1 and JAK-2 were overexpressed (Fig. 5C). Recently, we demonstrated that the oncogenic tyrosine kinase receptor MEN2A induces STAT3 transactivation in the absence of J AK tyrosine kinase activity [30]. As an alternative way to activate
STAT3 independent of the LIF receptor components, MEN2A was overexpressed in N tera-2/D1 EC cells and STAT3 transactivation was determined by transient cotransfection of the IRE-LUC reporter. As depicted in Fig. 5D, overexpression of MEN2A induced a 3-fold induction of IRE transactivation, while MEN2A induced an 11-fold induction in the presence of overexpressed STAT3. Overexpression of STAT3 alone did not significantly enhance IRE transactivation. Taken together, these data strongly suggest that the perturbation in LIF-STAT3 signaling in human N tera-2/D1 EC cells lies upstream of STAT3.

\section{Constitutive SOCS-1 Expression in Human N} Tera-2/D1 EC Cells

STAT3 signal transduction is downregulated by a family of negative feedback proteins, which includes SOCS-1 and SOCS-3. Since all the required signal transduction components which are required to mediate LIF signal transduction are expressed in $\mathrm{N}$ tera2/D1 EC cells, it was speculated that a constitutive activation of negative feedback proteins might prevent LIF signaling in N tera-2/D1 EC cells. Total RNA from 
A

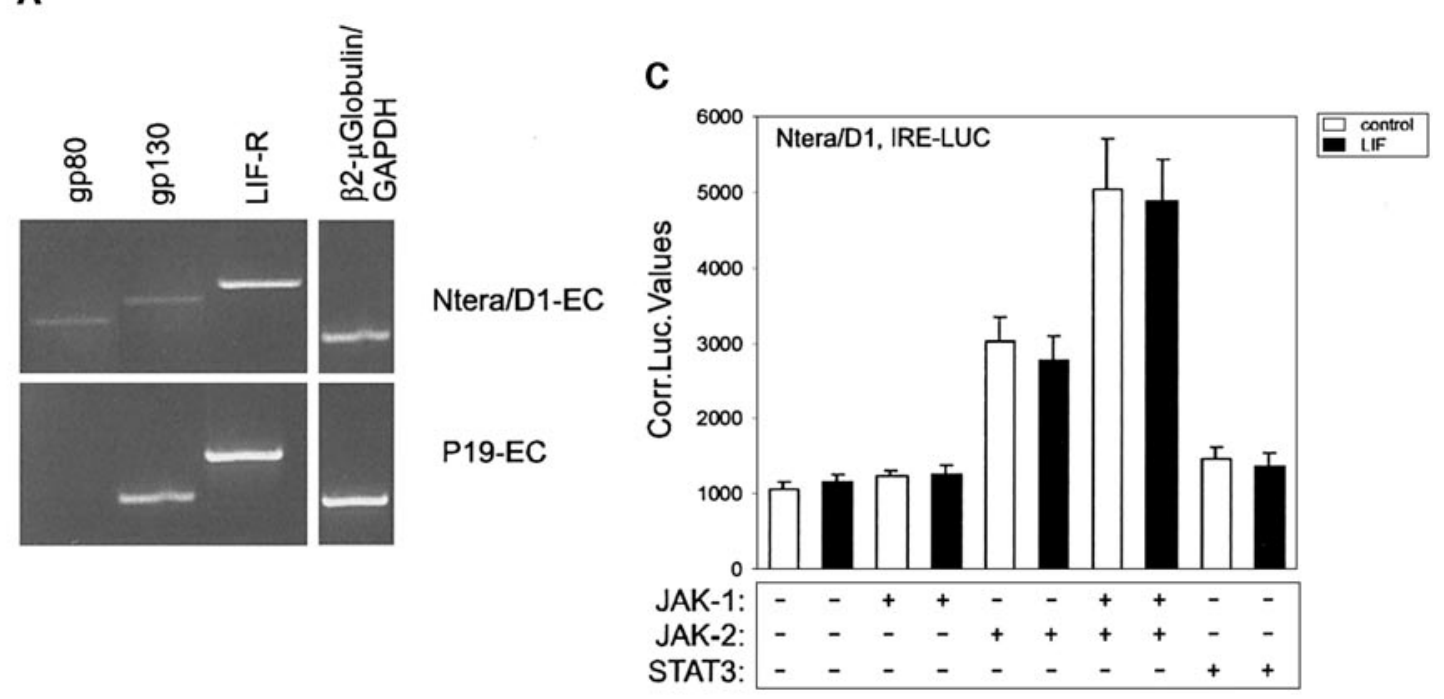

B
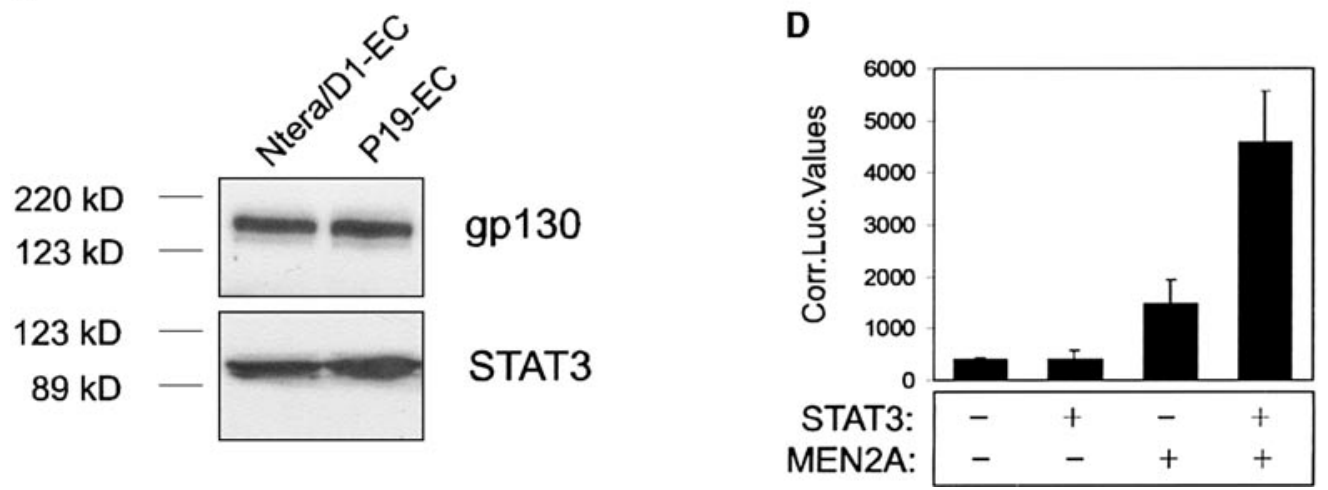

FIG. 5. The expression of receptor components in P19 EC and N tera-2/D1 EC cells. (A) Total RNA was isolated from N tera-2/D1 EC cells or P19 EC cells and RT-PCRs were performed as described under Materials and Methods using primers for gp80, gp130, or LIF-R. As an internal control RT-PCRs were performed using primers for GAPDH or $\beta 2$-microglobulin. (B) Total cell extracts of N tera-2/D1 EC or P19 EC cells were Western blotted using antibodies recognizing gp130 or STAT3. (C) N tera-2/D1 EC cells were transiently transfected with the IRE-LUC reporter and cells were cotransfected with $2.5 \mu \mathrm{g}$ expression vectors encoding J AK 1 , J AK 2, or STAT3 as indicated. Cells were stimulated with $10^{6}$ units/ml human LIF. (D) Transient transfection assays as in C, but now N tera-2/D1 cells were transfected with expression vectors for STAT3 and/or MEN2A as indicated.

unstimulated and hLIF-stimulated cells was isolated for RT-PCR reactions using primers for SOCS-1 and SOCS-3. In unstimulated N tera-2/D1 EC cells, high levels of SOCS-1 expression were observed, which were not further upregulated upon hLIF stimulation (Fig. 6). Both in unstimulated and in hLIF-stimulated cells, no SOCS-3 expression was observed (Fig. 6). Treatment of P19 EC cells with hLIF for either 1 or $2 \mathrm{~h}$ led to an increase in SOCS-3 mRNA levels while no upregulation of SOCS-1 was observed (Fig. 6). $\beta 2$-Microglobulin and GAPDH RNA levels were not affected by LIF treatment of N tera-2/D1 EC and P19 EC cells, respectively. These data indicate that in $\mathrm{N}$ tera-2/D1 EC cells, a negative feedback loop is constitutively activated, in contrast to P19 EC cells.
The gp130 Receptor Is Not Activated by LIF in Human N Tera-2/D1 EC Cells

The expression of SOCS proteins prevents J AK-mediated phosphorylation of tyrosine residues of cytokine receptors. Therefore, it was determined whether the gp130 receptor was phosphorylated on tyrosine residues in response to LIF. Gp130 was immunoprecipitated from unstimulated and hLIF-stimulated $\mathrm{N}$ tera2/D1 EC and P19 EC cells, and precipitates were Western blotted using antibodies recognizing phosphorylated tyrosine residues (PY-99). As depicted in Fig. 7, no gp130 phosphorylation was observed in either unstimulated or hLIF-stimulated N tera-2/D1 EC cells, while hLIF did induce gp130 tyrosine phosphor- 


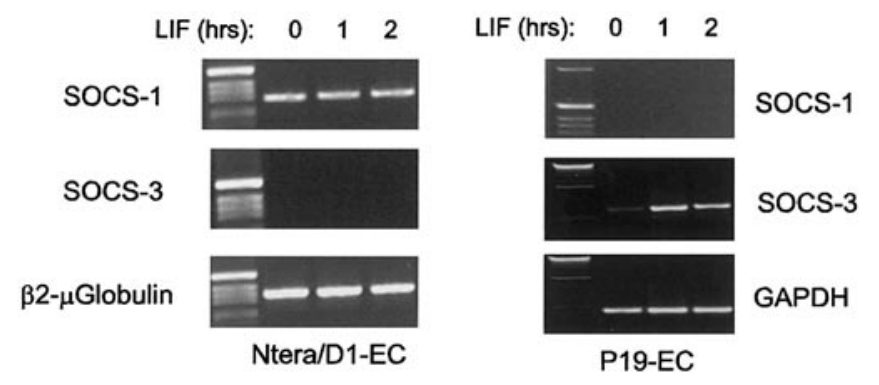

FIG. 6. SOCS-1 is constitutively expressed in N tera-2/D1 EC cells. N tera-2/D1 EC cells (left) or P19 EC cells (right) were stimulated with $10^{6}$ units/ml human LIF for 1 or $2 \mathrm{~h}$ or left unstimulated as indicated. Total RNA was isolated and RT-PCRs were performed as described under Materials and Methods using primers for SOCS1 or SOCS-3. As an internal control, RT-PCRs were performed using primers for $\beta 2$-microglobulin (left) or GAPDH (right).

ylation in P19 EC cells. As a control, gp130 was immunoprecipitated from IL-6-stimulated HepG2 cells. IL-6 stimulation resulted in a strong tyrosine phosphorylation of the gp130 receptor within $10 \mathrm{~min}$ (Fig. 7). These results indicate that LIF does not activate the gp130 receptor in N tera-2/D1 EC cells, although gp130 is properly expressed, implying that STAT3 is not activated in human $\mathrm{N}$ tera-2/D1 cells due to the absence of activated gp130.

\section{DISCUSSION}

Recent advances in mouse embryonal stem cell biology have elucidated the critical roles of LIF and STAT3 for stem cell self-renewal and the maintenance of pluripotency. As a consequence, mouse ES cells can now be cultured in the absence of embryonal fibroblast feeder-cell layers when LIF is administered to the medium [5-8]. However, human ES cells do not respond to LIF in a similar fashion and still require feeder-cell layers for support [27]. EC cells provide a tool for analyzing the mechanisms that control differentiation during embryonal development [23]. They express markers that are also expressed in ES cells, including Oct-4, alkaline phosphatase, and stage-specific embryonic antigens 3 and 4 , but not 1 , as mouse EC and ES cells, and can be differentiated into cell types of all three germ layers [23]. Here, we set out to study the signal transduction cascades that are initiated by LIF in mouse and human embryonal carcinoma cells (P19 EC and N tera-2/D1 EC cells). We report that LIF induces both ERK-1 and STAT3 activation in P19 EC cells, but that LIF-induced proliferation depends on ERK activity only. In contrast, LIF does not activate STAT3, ERK, or the gp130 receptor in N tera-2/D1 EC cells, although required signal transduction components are expressed. The negative feedback protein SOCS-1 is highly expressed in human $\mathrm{N}$ tera-2/D1 EC cells, which might prevent LIF-induced STAT3 signal transduction.

In P19 EC cells, LIF induces STAT3 tyrosine 705 phosphorylation within $15 \mathrm{~min}$. This results in rapid STAT3 dimerization, nuclear translocation, and DNA binding (data not shown) as well as in target gene expression as determined by STAT3 reporter assays.

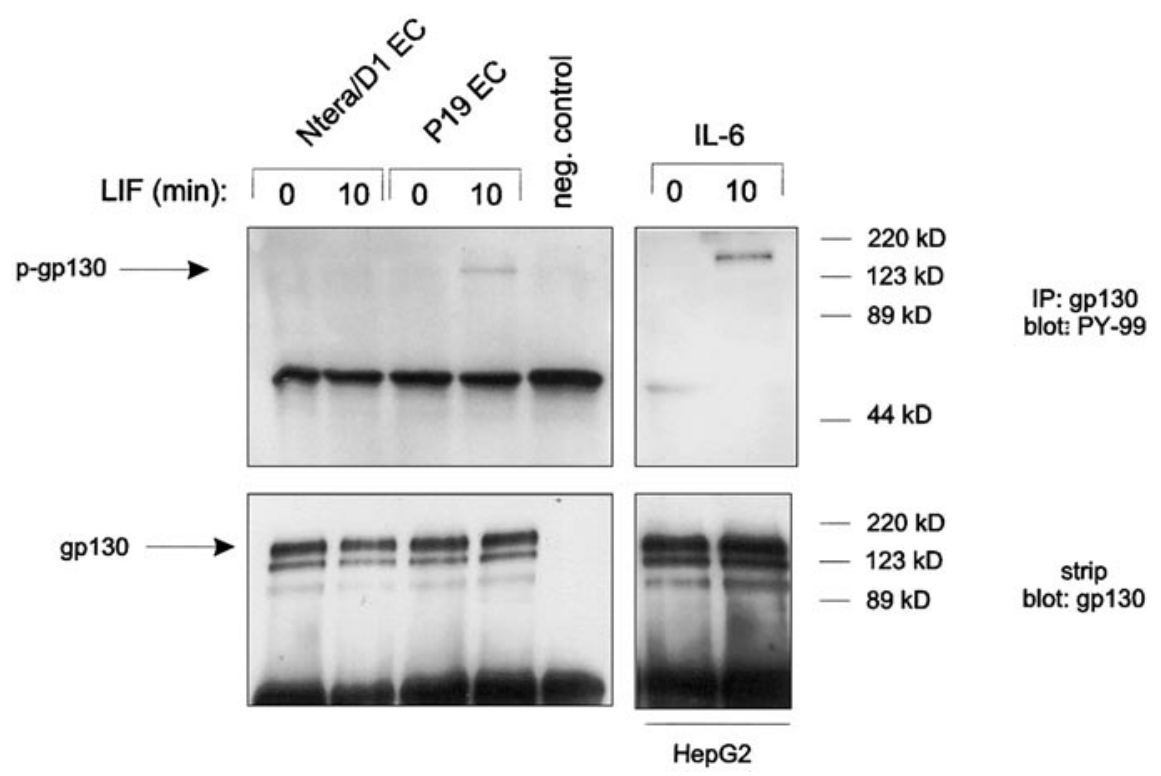

FIG. 7. The gp130 receptor is not activated by LIF in human $N$ tera-2/D1 EC cells. Gp130 was immunoprecipitated from hLIF-stimulated $\mathrm{N}$ tera-2/D1 EC cells, P19 EC cells (10 min) and IL-6-stimulated HepG2 cells (10 min) and immunoprecipitates were Western blotted using antibodies recognizing phosphorylated tyrosine residues (PY-99). As a control, blots were stripped and reprobed with anti-gp130 antibodies (bottom panels). As a negative control, lysates were incubated with protein A-Sepharose beads in the absence of antibodies. 
Besides a LIF-induced activation of synthetic IRE-reporters, we also observed that LIF induces STAT3mediated transactivation of the ICAM-1 promoter, in agreement with data published by Hocke et al. [34]. The LIF-induced STAT3 transactivation is completely dependent on J AK2 kinase activity, since inhibition of J AK2 by using the inhibitor AG490 completely abolished the LIF-induced STAT3 tyr705 phosphorylation and transactivation. In contrast to LIF, IL-6 does not activate STAT3 in P19 EC cells. Since gp80 RNA is not expressed in P19 EC cells we conclude that IL -6 does not signal due to the absence of the I $L-6$ binding receptor. These results are in agreement with previously published data in which addition of the soluble IL-6 receptor was required to initiate IL-6 signal transduction in mouse ES cells [35].

LIF induces proliferation of P19 EC cells as determined by MTC assays. However, the LIF-induced STAT3 activation is not coupled to proliferation since inhibition of STAT3 activity by using the J AK 2 inhibitor AG490 did not affect LIF-induced proliferation of P19 EC cells. In mouse ES cells, it has been demonstrated that ES cell growth is independent of the LIF concentration [36]. Further experiments indicated that similar proportions of ES cells were in the $G 2, M$, or $S$ phase, regardless of whether the cultures were supplemented with $500 \mathrm{pM} \mathrm{LIF}$ or $1 \mu \mathrm{g} / \mathrm{ml} \alpha$-LIF to inhibit low levels of LIF present in serum [36]. However, the LIF concentration appeared to be important in determining the probability of an ES cell undergoing a selfrenewal versus a differentiation division. Similar observations have been published by Raz et al. who demonstrate that LIF inhibits ES cell differentiation rather than potentiating the proliferation of ES cells $[8,23]$. In contrast, in EC cells we find that LIF does induce proliferation, and that this is dependent on activation of the ERK pathway. LIF induces activation of ERK-1 phosphorylation within 15 min upon stimulation, which is blocked by the MEK inhibitor PD98059. Treatment of P19 EC cells with the inhibitor PD98059 completely abolished LIF-induced proliferation. Since PD98059 did not affect LIF-induced STAT3 tyr705 phosphorylation, we conclude that LIF-induced proliferation of P19 EC cells is independent of STAT3 signaling but requires activation of ERK.

A peculiar finding was observed when P19 EC cells were treated with the MEK inhibitor PD98059. LIFinduced STAT3 tyr705 phosphorylation was not affected by PD98059 and the LIF-induced fold induction of STAT3 transactivation was still approximately 3-fold, comparable to the fold induction that was observed in cells that were not treated with PD98059. However, the absolute levels of reporter activity were significantly increased. Similar results were obtained in studies using the ICAM-luciferase or IRE-mut-luciferase reporter, in which the STAT3 binding sites are mutated, indicating that inhibition of ERK activity does not directly affect STAT3 signal transduction. Further experiments are required to resolve these issues, but similar effects of PD98059 on gene transcription were observed in N tera-2/D1 EC cells. Burdon et al. demonstrated that mouse stem cell colonies formed at 100-fold lower LIF concentrations when the gp130 receptor was mutated at position 118 [35]. This mutant is no longer capable of binding SHP-2 and fails to activate the ERK signal transduction cascade. In the absence of ERK signaling, a sustained STAT3 activation was observed in response to gp130 receptor activation. It was concluded that ERK activation is not required for self-renewal of mouse ES cells and that the growth of undifferentiated ES cells was even enhanced in the presence of PD98059 [35]. Possibly, the effects of PD98059 on ES growth are not directly related to enhanced activation of STAT3 but rather related to enhanced effects of PD98059 on gene transcription in general, possibly by modulating the activity of the basal RNA polymerase transcription machinery.

In human N tera-2/D1 EC cells, we did not observe a LIF-induced STAT3 or ERK activation. STAT3 and ERK were not phosphorylated in response to LIF, and no LI F-induced STAT3 transactivation was detected in reporter assays using IRE-luciferase or ICAM-Iuciferase reporters. Also, no effects of LIF could be detected on the proliferation of $\mathrm{N}$ tera-2/D1 EC cells in MTC assays. We can exclude the possibility that these observation are due to differences in species-specific LIF-receptor interactions, since neither murine nor human LIF activated STAT3 in N tera-2/D1 EC cells, while LIF from both species induced STAT3 transactivation to similar levels in P19 EC cells. Also, no IL-6induced STAT3 phosphorylation or STAT3 transactivation was observed in N tera-2/D1 EC cells, although all the required receptor components for IL-6 and LIF signal transduction, gp80, gp130, and the LIFR, were readily detected in RT-PCR assays or by Western blotting. Although further experiments are required to determine whether these proteins are also functional, these results suggest that signaling is disturbed downstream of the receptor components. Reporter studies in which J AK 1, J AK2, or STAT3 were overexpressed revealed that neither of these could reconstitute LIFinduced STAT3 signal transduction, indicating that possible low endogenous expression levels or acquired mutations in these signal transduction components cannot account for the observed results. These results were further underscored by the fact that overexpression of the oncogenic tyrosine kinase receptor MEN2A, which activates STAT3 in a J AK-2-independent manner but requires its intrinsic tyrosine kinase domain [30], does activate STAT3 in N tera-2/D1 EC cells. These observations imply that disturbed LIF-STAT3 
signaling in human $\mathrm{N}$ tera-2/D1 EC cells involves a disturbed activation of STAT3-upstream components.

TheSOCS family of negative feedback proteins fulfill crucial roles in the downregulation of STAT signal transduction [reviewed in 22, 37]. While in P19 EC cells no basal levels of SOCS-1 or SOCS-3 RNA were detected, SOCS-3 was upregulated within $1 \mathrm{~h}$ after LIF stimulation. In contrast, high basal levels of SOCS-1 RNA were detected in unstimulated N tera-2/D1 EC cells, which were not further upregulated in response to LIF. Also, no increase in SOCS-3 expression was observed after either 1 or $2 \mathrm{~h}$ of LIF stimulation. Although these data will have to be confirmed on the level of SOCS protein expression, these results suggest that LIF-STAT3 signaling might be disturbed due to a constitutive activation of a negative feedback loop that results in the expression of SOCS-1. The expression of SOCS proteins prevent a J AK-mediated phosphorylation of tyrosine residues of cytokine receptors. Indeed, in N tera-2/D1 EC cells no LIF-induced tyrosine phosphorylation of the gp130 receptor was observed, while gp130 was readily phosphorylated in P19 EC cells in response to LIF or in HepG2 cells in response to IL-6. Therefore, our data imply that LIF does not activate STAT3 in human N tera-2/D1 EC cells due to a constitutive expression of SOCS-1, which prevents LIF-induced tyrosine phosphorylation of the gp130 receptor.

In mouse ES cells, Duval et al. reported that the expression of SOCS-3, but not of SOCS-1 or SOCS-2, was stimulated in the presence of LIF [38], similar to what we observe in P19 EC cells. Furthermore, the uncontrolled overexpression of SOCS-1 and SOCS-3 led to repression of LIF-dependent transcription and severely reduced cell viability, suggesting that a disturbance of a well-balanced SOCS protein content has adverse effects on cell survival [38]. Future experiments to interfere with endogenous SOCS-1 expression will reveal whether the constitutive expression of SOCS-1 indeed prevents LIF signaling in human EC cells. Furthermore, experiments are under way to determine whether SOCS-1 is also constitutively expressed in human ES cells. When this is indeed the case, it might become feasible to culture human ES cells in the absence of feeder cells by using strategies that prevent the endogenous expression of SOCS-1.

This work was supported by grants from the Dutch Cancer Foundation (RUG 96-1217 and RUG 00-2316).

\section{REFERENCES}

1. Evans, M. J ., and Kaufman, M. H. (1981). Establishment in culture of pluripotential cells from mouse embryos. Nature 292, 154-156.

2. Martin, G. R. (1981). I solation of a pluripotent cell line from early mouse embryos cultured in medium conditioned by ter- atocarcinoma stem cells. Proc. Natl. Acad. Sci. USA 78, 76347638.

3. Itskovitz-Eldor, J., Schuldiner, M., Karsenti, D., Eden, A., Yanuka, O., Amit, M., Soreq, H., and Benvenisty, N. (2000). Differentiation of human embryonal stem cells into embryoid bodies compromising the three embryonal germ layers. Mol. Med. 6, 88-95.

4. Reubinoff, B. E., Pera, M. F., Fong, C. Y., Trounson, A., and Bongso, A. (2000). Embryonal stem cell lines from human blastocysts: Somatic differentiation in vitro. Nature Biotechnol. 18, 399- 404.

5. Boeuf, H., Hauss, C., Graeve, F. D., Baran, N., and Kedinger, C. (1997). Leukemia inhibitory factor-dependent transcriptional activation in embryonal stem cells. J . Cell Biol . 138, 1207-1217.

6. Niwa, H., Burdon, T., Chambers, I., and Smith, A. (1998). Self-renewal of pluripotent embryonal stem cells is mediated via activation of STAT3. Genes Dev. 12, 2048-2060.

7. Matsuda, T., Nakamura, T., Nakao, K., Arai, T., Katsuki, M., Heike, T., and Yokota, T. (1999). STAT3 activation is sufficient to maintain an undifferentiated state of mouse embryonal stem cells. EMBO J . 18, 4261- 4269.

8. Raz, R., Lee, C. K., Cannizzaro, L. A., d'Eustachio, P., and Levy, D. E. (1999). Essential role of STAT3 for embryonal stem cell pluripotency. Proc. Natl. Acad. Sci. USA 96, 2846-2851.

9. Heinrich, P. C., Behrmann, I., Muller, N. G., Schaper, F., and Graeve, L. (1998). Interleukin-6-type cytokine signalling through the gp130/J ak/STAT pathway. Biochem. J . 334, 297314.

10. Gearing, D. P., Comeau, M. R., Friend, D. J., Gimpel, S. D., Thut, C. J ., McGourty, J ., Brasher, K. K., King, J . A., Gillis, S., Mosley, B., et al. (1992). The IL-6 signal transducer, gp130: An oncostatin $M$ receptor and affinity converter for the LIF receptor. Science 255, 1434-1437.

11. Stahl, N., Boulton, T. G., Farruggella, T., Ip, N. Y., Davis, S., Witthuhn, B. A., Quelle, F. W., Silvennoinen, O., Barbieri, G., and Pellegrini, S. (1994). Association and activation of J ak-Tyk kinases by CNTF-LIF-OSM-IL- 6 beta receptor components. Science 263, 92-95.

12. Schindler, C., and Darnell, J. E. (1995). Transcriptional responses to polypeptide ligands: The J AK-STAT pathway. Annu. Rev. Biochem. 64, 621-651.

13. Fukada, T., Hibi, M., Yamanaka, Y., Takahashi, T. M., Fujitani, Y., Yamaguchi, T., Nakajima, K., and Hirano, T. (1996). Two signals are necessary for cell proliferation induced by a cytokine receptor gp130: Involvement of STAT3 in anti-apoptosis. Immunity 5, 449-460.

14. Nishida, K., Y oshida, Y., Itoh, M., Fukada, T., Ohtani, T., Shirogane, T., Atsumi, T., Takahashi-Tezuka, M., Ishihara, K., Hibi, M., and Hirano, T. (1999). Gab-family adapter proteins act downstream of cytokine and growth factor receptors and $\mathrm{T}$ - and B-cell antigen receptors. Blood 93, 1809-1816.

15. Takahashi-Tezuka, M., Yoshida, Y., Fukada, T., Ohtani, T., Yamanaka, Y., Nishida, K., Nakajima, K., Hibi, M., and Hirano, T. (1998). Gabl acts as an adapter molecule linking the cytokine receptor gp130 to ERK mitogen-activated protein kinase. Mol. Cell. Biol. 18, 4109- 4117.

16. Endo, T. A., Masuhara, M., Yokouchi, M., Suzuki, R., Sakamoto, H., Mitsui, K., Matsumoto, A., Tanimura, S., Ohtsubo, M., Misawa, H., Miyazaki, T., Leonor, N., Taniguchi, T., Fujita, T., Kanakura, Y., Komiya, S., and Yoshimura, A. (1997). A new protein containing an $\mathrm{SH} 2$ domain that inhibits J AK kinases. Nature 387, 921-924.

17. Naka, T., Narazaki, M., Hirata, M., Matsumoto, T., Minamoto, S., Aono, A., Nishimoto, N., Kajita, T., Taga, T., Yoshizaki, K., 
Akira, S., and Kishimoto, T. (1997). Structure and function of a new STAT-induced STAT inhibitor. Nature 387, 924-929.

18. Starr, R., Willson, T. A., Viney, E. M., Murray, L. J ., Rayner, J . R., J enkins, B. J ., Gonda, T. J ., Alexander, W. S., Metcalf, D., Nicola, N. A., and Hilton, D. J . (1997). A family of cytokineinducible inhibitors of signalling. Nature 387, 917-921.

19. Hilton, D. J ., Richardson, R. T., Alexander, W. S., Viney, E. M., Willson, T. A., Sprigg, N. S., Starr, R., Nicholson, S. E., Metcalf, D., and Nicola, N. A. (1998). Twenty proteins containing a C-terminal SOCS box form five structural classes. Proc. Natl. Acad. Sci. USA 95, 114-119.

20. Nicholson, S. E., Willson, T. A., Farley, A., Starr, R., Zhang, J . G., Baca, M., Alexander, W. S., Metcalf, D., Hilton, D. J ., and Nicola, N. A. (1999). Mutational analyses of the SOCS proteins suggest a dual domain requirement but distinct mechanisms for inhibition of LIF and IL-6 signal transduction. EMBO J . 18, 375-385.

21. Song, M. M., and Shuai, K. (1998). The suppressor of cytokine signaling (SOCS) 1 and SOCS3 but not SOCS2 proteins inhibit interferon-mediated antiviral and antiproliferative activities. J . Biol. Chem. 273, 35056-35062.

22. Starr, R., and Hilton, D. J . (1999). Negative regulation of the J AK/STAT pathway. Bioessays 21, 47-52.

23. Andrews, P. W. (1998). Teratocarcinomas and human embryology: Pluripotent human EC cell lines [Review article]. APMIS 106, 158-167.

24. Thomson, J. A., Itskovitz-Eldor, J., Shapiro, S. S., Waknitz, M. A., Swiergiel, J . J ., Marshall, V. S., and J ones, J . M. (1998). Embryonal stem cell lines derived from human blastocysts. Science 282, 1145-1147.

25. Lehtonen, E., Laasonen A., and Tienari, J . (1989) Teratocarcinoma stem cells as a model for differentiation in the mouse embryo. Int. J . Dev. Biol. 33, 105-115.

26. McBurney, M. W. (1993). P19 embryo carcinoma cells. Int. J . Dev. Biol. 37, 135-140.

27. Pera, M. F., Reubinoff, B., and Trounson, A. (2000). Human embryonal stem cells. J . Cell Sci. 113(Pt. 1), 5-10.

28. Schuringa, J . J ., J onk, L. J . C., Dokter, W. H. A., Vellenga, E., and Kruijer, W. (2000). IL-6 induced STAT3 transactivation and Serine 727 phosphorylation involves Vav, Rac, and SEK/
MKK-4 as signal transduction components. Biochem. J . 347, 89-96.

29. Caldenhoven, E., Coffer, P., Yuan, J ., Van-de-Stolpe, A., Horn, F., Kruijer, W., and Van-der-Saag, P. T. (1994). Stimulation of the human intercellular adhesion molecule-1 promoter by interleukin- 6 and interferon-gamma involves binding of distinct factors to a palindromic response element. J . Biol. Chem. 269, 21146-21154.

30. Schuringa, J.J., Wojtachnio, K., Hagens, W., Vellenga, E., Buys, C. H., Hofstra, R., and Kruijer, W. (2001). MEN2A induces cellular transformation by activation of STAT3. Oncogene 20, 5350-5358.

31. Graham, F. L., and Van der Eb, A. J . (1973). Transfection of mamalian cells using calcium phosphate. Mol. Cell. Biol. 2, 607- 616.

32. Brasier, A. R., Tate, J . E., and Habener, J . F. (1989). Optimized use of the firefly luciferase assay as a reporter gene in mammalian cell lines. Biotechniques 7, 1116-1122.

33. Delegeane, A. M., Ferland, L. H., and Mellon, P. L. (1987). Tissue-specific enhancer of the human glycoprotein hormone alpha-subunit gene: Dependence on cyclic AMP-inducible elements. Mol. Cell. Biol. 7, 3994-4002.

34. Hocke, G. M., Cui, M. Z., and Fey, G. H. (1995). The LIF response element of the alpha 2 macroglobulin gene confers LIF-induced transcriptional activation in embryonal stem cells. Cytokine 7, 491-502.

35. Burdon, T., Stracey, C., Chambers, I., Nichols, J ., and Smith, A. (1999). Suppression of SHP-2 and ERK signalling promotes self-renewal of mouse embryonal stem cells. Dev. Biol. 210, 30- 43.

36. Zandstra, P. W., Le, H. V., Daley, G. Q., Griffith, L. G., and Lauffenburger, D. A. (2000). Leukemia inhibitory factor (LIF) concentration modulates embryonal stem cell self-renewal and differentiation independently of proliferation. Biotechnol. Bioeng. 69, 607- 617.

37. Nicola, N. A., and Greenhalgh, C. J . (2000). The suppressors of cytokine signaling (SOCS) proteins: Important feedback inhibitors of cytokine action. Exp. Hematol. 28, 1105-1112.

38. Duval, D., Reinhardt, B., Kedinger, C., and Boeuf, H. (2000). Role of suppressors of cytokine signaling (Socs) in leukemia inhibitory factor (LIF) -dependent embryonal stem cell survival. FASEB J . 14, 1577-1584.

Received September 21, 2001

Revised version received November 22, 2001

Published online J anuary 22, 2002 